\title{
Versatility of the Superior Lateral Genicular Artery Perforator Flap for Coverage of Soft Tissue Defects Around the Knee
}

\author{
SHERIF M. ELKASHTY, M.D.; MOHAMED ABOSHABAN, M.D. and AHMED A. TAALAB, M.D. \\ The Department of Plastic and Reconstructive Surgery, Faculty of Medicine, Menoufia University, Menofia, Egypt
}

\begin{abstract}
Background: Coverage of soft tissue defects around the knee is a reconstructive challenge. The superior lateral genicular artery perforator (SLGAp) flap provides an excellent alternative to muscle and musclocutaneous flaps with less morbidity, for coverage of these defects, especially when the gastrocnemius muscle flap is not available. The vascular basis of this flap, SLGA perforators, have consistent and reliable anatomy.
\end{abstract}

Aim of the Study: This study is a retrospective analysis of our clinical experience with the superior lateral genicular artery perforator flap for coverage of soft tissue defects of the knee.

Patients and Methods: This study was done at The Department of Plastic and Reconstructive Surgery, Menofia University Hospitals, from January 2017 to November 2018, with follow-up period of 12 months. It included 7 patients, 6 males, and 1 female, with their age ranged from 19 to 46 years. The around-knee defects were due to different etiologies, 1 patient due to shotgun, 1 patient with post-burn contracture of the lateral popliteal fossa, and 5 patients with traffic accidents. The defects were reconstructed by the superior lateral genicular artery perforator (SLGAp) flap.

Results: All flaps survived, except in 2 cases, 1 case managed conservatively and healed well, and in the second, the necrotic part was debrided and skin grafted. The donor site was closed primarily in 2 cases and skin grafted in 5 cases. Skin graft take was perfect, except in 3 cases with minor patches of graft loss and healed spontaneously. Infection around the defect site occurred in 2 cases and managed conservatively. The flap provided stable and durable coverage with minimal donor site morbidity and no functional loss.

Conclusion: The superior lateral genicular artery perforator flap is a versatile option for reconstruction of soft tissue defects around the knee or popliteal fossa. The anatomy of the flap perforators is consistent and reliable. The flap is thin, pliable and provides stable coverage with adequate match of the surroundings. Donor site morbidity is minimal with no functional loss.

Key Words: Superior lateral genicular artery - Knee defects - Perforator flap.

\section{INTRODUCTION}

Soft tissue defects around the knee represent a reconstructive challenge. These may occur due to different causes as traffic accidents, shotgun, postburn contracture release, post- tumor excision, etc. There are many reconstructive options for covering soft tissue defects around the knee, including several muscle flaps, fasciocutaneous flaps, and free flaps, each with its inherent advantages and disadvantages [1-10].

The superior lateral genicular artery perforator (SLGAp) flap is a fasciocutaneous flap based on the cutaneous perforators of the superior lateral genicular artery (SLGA). Many authors recommended its use and documented its versatile features, as being thin, pliable, matching like with like, etc., for soft issue reconstruction of defects around the knee, especially when the gastrocnemius muscle is not available for use, or avoided because of its bulk and consequent functional deficit [11-18].

In 1989, Laitung found that $86 \%$ of posterolateral thigh flaps were supplied by the lateral superior genicular artery [11]. Hayashi and Maruyama, in 1990, after a cadaveric study of 10 cadavers, found the (SLGAp) flap to be reliable in 3 cases [12]. In 1994, Spokevicius and Jankauskas had done an anatomical study with 9 fresh cadavers and 3 clinical applications of the (SLGAp) flap, as a free flap in 2 cases and a pedicled flap in 1 case [13]. Saint-Cyr et al., in 2011, after a cadaveric study of 28 limbs, used the (SLGAp) flap in 2 patients [16]. In 2011, Wiedner et al., used the (SLGAp) flap in 6 patients with adequate results [17]. In 2018, Zhihua Li BS and colleagues used the (SLGAp) flap in 5 cases [18]. 
The aim of this study is to evaluate the versatility of the superior lateral genicular perforator flap for coverage of soft tissue defects around the knee, resulting from different etiologies, and to determine flap advantages and disadvantages.

\section{Relevant surgical anatomy:}

Hayashi and Maruyama, in 1990, on the basis of 10 cadaver dissections, found that SLGA was identified in all 10 cadavers, and originated from popliteal artery in 8 limbs, and from the sural artery in 2 limbs. The SLGA travelled through the intermuscular septum between the vastus lateralis and short head of biceps femoris, and then penetrated the deep fascia just proximal to the lateral condyle of femur, averaging 3 to $8 \mathrm{~cm}$. from the plane of knee joint [12].

Saint-Cyr et al., in 2011, on the basis of 28 cadaveric lower limb dissections and injection studies, found that, between 1 to 3 SLGA perforators in each lower limb, with median of 2 perforators in each limb. All the perforators were found within $5 \mathrm{~cm}$. laterally and $7 \mathrm{~cm}$. proximally from the superolateral patella. Contrast injection into the SLGA perforators demonstrated its perforasome to be limited to the lower half of the lateral thigh, with absence of contrast below the patella, suggesting that the direction of flow was away from the knee [16].

\section{PATIENTS AND METHODS}

This study was done at The Department of Plastic and Reconstructive Surgery, Menofia University Hospitals. It was done in the period from January 2017 to November 2018, with a followup period of an average 12 months. The study included 7 patients, 6 males and 1 female. Their age ranged from 19 to 46 years.

The soft tissue defects were located around the knee, at the anterior aspect in 5 patients, and at the lower lateral aspect in 1 patient, and at the lateral popliteal fossa in 1 patient. Regarding the etiology, 1 patient had shotgun injury, 5 patients had traffic accidents, and 1 patient had post-burn contracture of the lateral edge of popliteal fossa. Patients with associated bone fractures or vascular injury were managed first by orthopedic and vascular surgery and then, referred to our Department for coverage of soft tissue defects.

One patient had (presented in Fig. 1) shotgun injury, caused injury of popliteal artery distal to origin of SLGA perforators, injury of sural vascular pedicles of gastrocnemius muscle, and comminuted fracture of the tibia. Vascular surgeons repaired the popliteal artery by reversed saphenous vein graft and orthopedic surgeons applied external fixation to the tibial fracture. Orthopedic surgeons managed another patient with post RTA fracture of upper tibia, by intramedullary nail. Other remaining patients had no vascular nor bone injury.

Patients who had severe scarring or severe trauma at the site of the perforators were excluded as the pedicle may become impaired.

\section{Surgical technique:}

Pre-operative marking was done by identification of superior lateral genicular artery perforators by an $8 \mathrm{MHz}$ hand-held Doppler at the septum between the vastus lateralis and short head of biceps femoris, from the knee level proximally. The flap was marked from knee level up to midpoint between the femoral condyle and greater trochanter, with flap axis laying over that intermuscular septum, Fig. (1-A).

The procedures were done under spinal anesthesia with tourniquet control without exsanguination to easily detect the SLGA perforators. The anterior margin of the flap was incised down to deep fascia, with subfascial dissection towards the intermuscular septum to identify the SLGA perforators, Fig. (1$\mathrm{B}, \mathrm{C})$. We did not skeletonize the perforators, only dissect them to allow tension-free flap inset without perforator kinking or spasm. After dissection and isolation of the SLGA perforators, dissection continued in the subfascial plane towards the flap posterior margin, which was incised to completely island the flap, to be only hinged by the perforators, Fig. (1-D,E).

The tourniquet was deflated, flap viability was checked, and minor perforator was clamped, preserving only the dominant perforator. The perforator flap could be rotated 90 degrees counter-clockwise or clockwise to cover anterior surface of the knee or popliteal fossa, respectively, Fig. (1-F) or even rotated 180 degrees in a propeller fashion to cover the proximal one third of the leg, Fig. (1-G).

The SLGAp flap was transferred into the defect, with excision of any intervening skin bridge, with suction drain inserted on need. The flap was inset in a tension-free manner to avoid spasm or kinking of the pedicle and the flap donor site was either closed primarily or split-skin grafted, Fig. (1-H,I). 


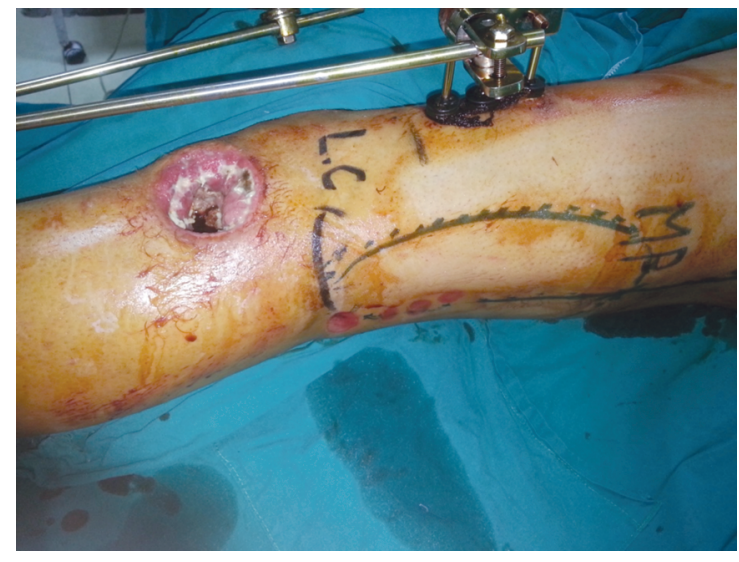

Fig. (1-A): Pre-operative photo of flap marking. Red dots demonstrate Doppler-detected perforators. LC stands for lateral condyle of femur. MP stands for mid-point between lateral condyle and greater trochanter. The wound caused by shotgun injury with injury of popliteal artery and gastrocnemius muscle.

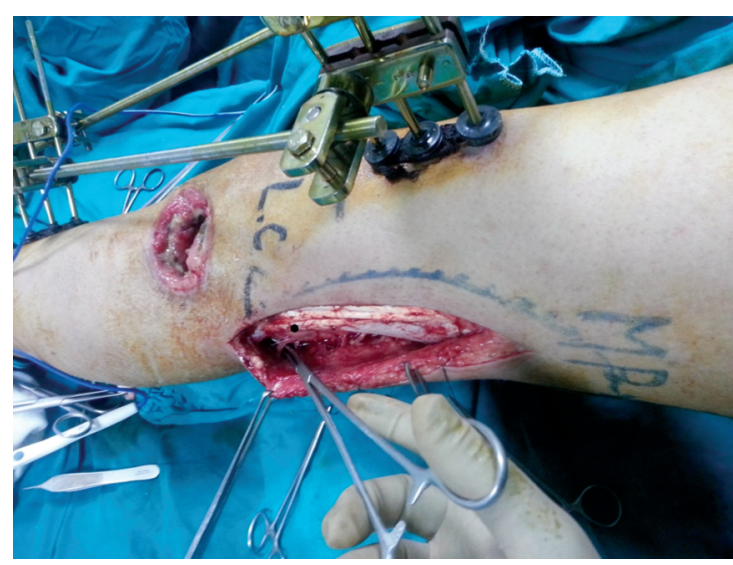

Fig. (1-C): A second perforator was dissected (shown over the tips of right angled clamp and marked by a black dot) in the septum between vastus lateralis and short head of biceps femoris.

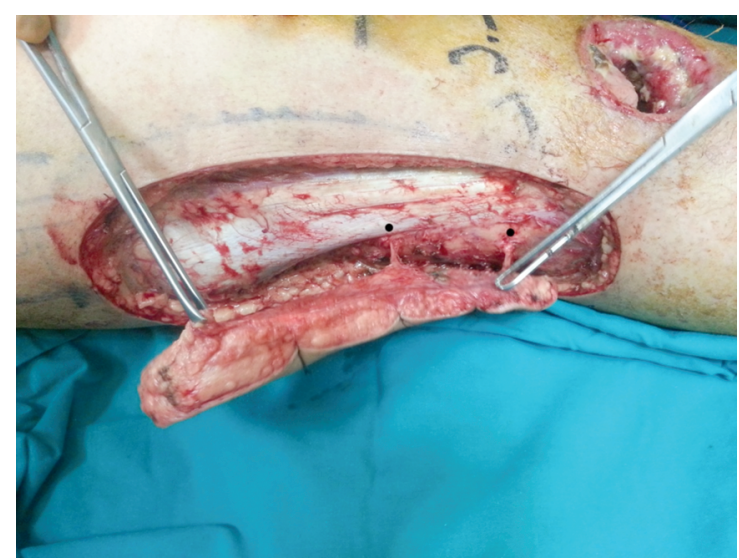

Fig. (1-E): The flap totally islanded on 2 perforators, marked by black dots.

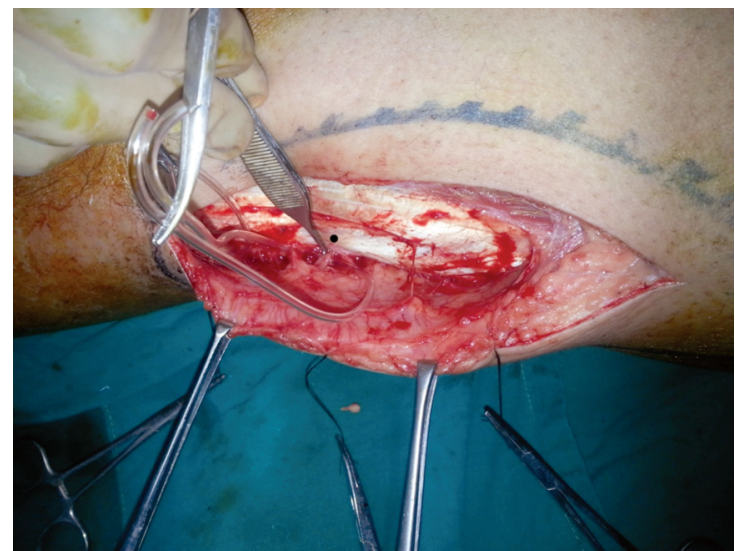

Fig. (1-B): Subfascial dissection of the anterior margin with one perforator isolated at the tip of forceps (marked by a black dot) in the septum between vastus lateralis and short head of biceps femoris.

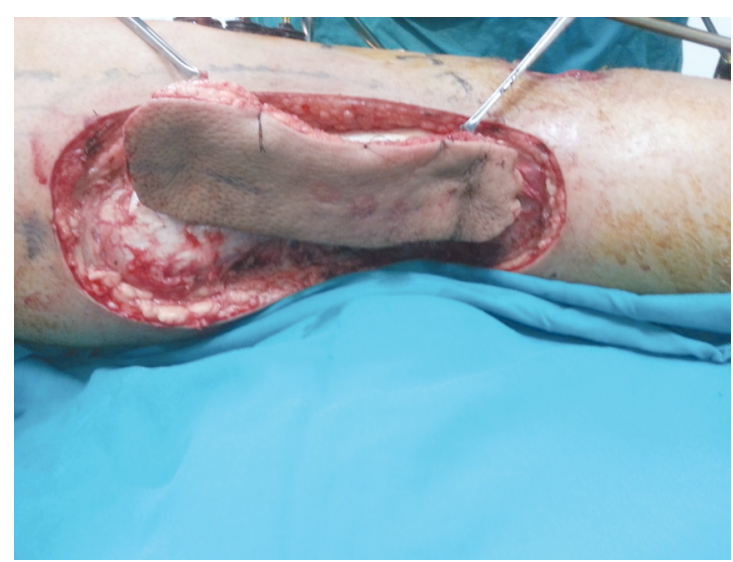

Fig. (1-D): The SLGA perforator flap totally islanded, only hinged on the 2 perforators.

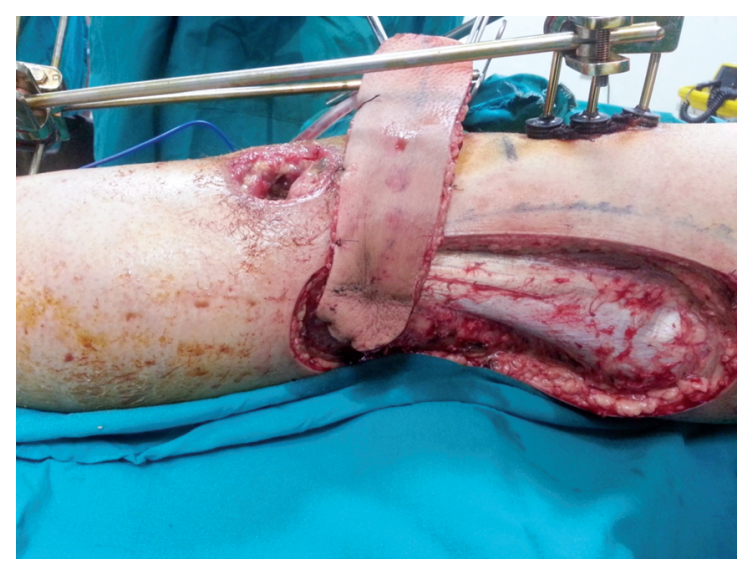

Fig. (1-F): The SLGA perforator flap rotated 90 degrees counter-clockwise to reconstruct anterior surface of knee. 


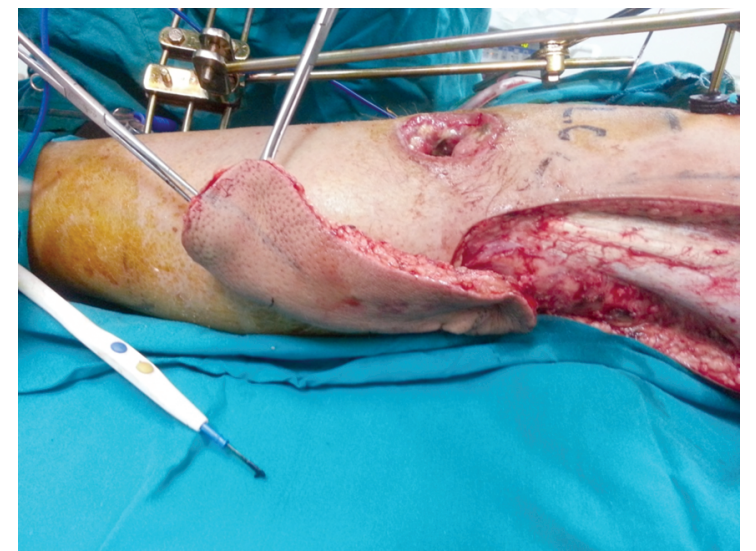

Fig. (1-G): The SLGA perforator flap could be rotated 180 degrees in a propeller fashion to reconstruct proximal one-third of the leg.

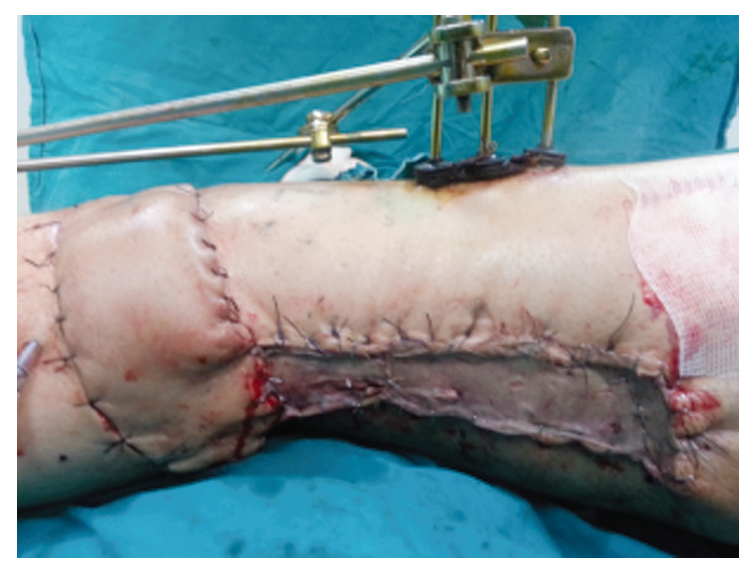

Fig. (1-I): SLGA perforator flap was inset and donor site was split skin-grafted.

\section{RESULTS}

This study included 7 patients, 6 males and 1 female, presented with defects around the knee, at the anterior aspect of the knee in 5 cases, at lower lateral aspect of the leg in 1 case, and at the lateral border of popliteal fossa after releasing post-burn contracture.

The etiology of the defects was due to shotgun wound in 1 case, traffic accident in 5 cases, and post-burn contracture of lateral popliteal fossa in one case.

The flap size ranged from $6 \times 11 \mathrm{~cm}$. to $10 \mathrm{x} 22 \mathrm{~cm}$, with 2 perforators in 5 SLGA flaps and 1 perforator in 2 flaps, located averagely from 5 to $10 \mathrm{~cm}$ proximal to knee level.

All flaps survived except in 2 cases, where 1 case had marginal flap necrosis at the edge farther from the pedicle, and managed conservatively with debridement and frequent dressings and healed spontaneously, Fig. (1-J). In the second case (Fig.

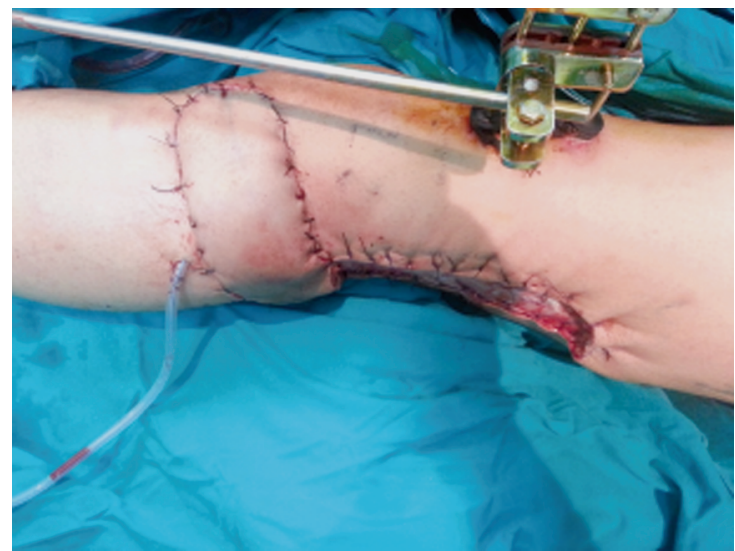

Fig. (1-H): SLGA perforator flap was inset into the defect and the flap donor site reduced in size before skin grafting.

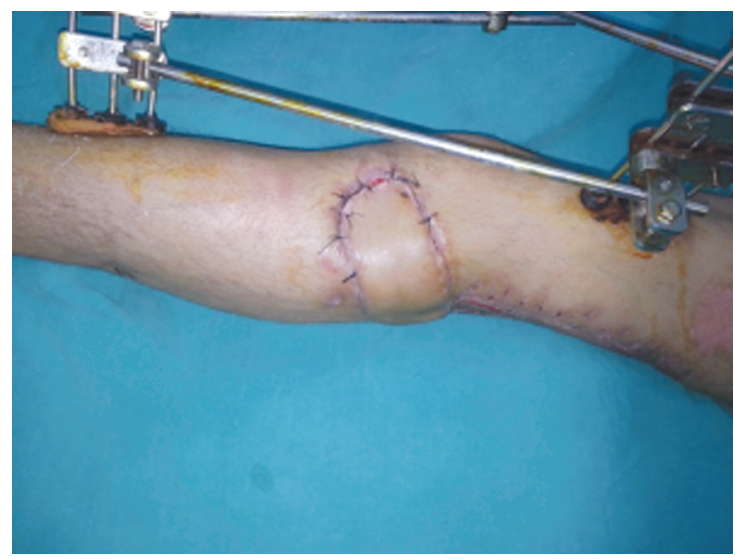

Fig. (1-J): A 6-week post-operative photo showed good healing of the flap, except small area at its most distal tip.

2A-E), the flap was used in a propeller fashion and had distal one-third necrosis, where the necrotic part was debrided and split-skin graft applied, Fig. (2-F,G).

The donor site was closed primarily in 2 cases, in which small flaps was used, up to $8 \mathrm{~cm}$ width. In the remaining 5 cases, split-skin graft was applied to the donor site. The take of the skin graft was good, except in 3 cases, where there were patchy graft losses, managed conservatively and healed spontaneously.

Infection at the original defect occurred in 2 cases and managed by culture-specific antibiotics and frequent dressing and healed uneventfully.

As regards the donor site morbidity, there was no functional deficit, with acceptable scar/graft appearance. The aesthetic appearance of the flap was satisfactory for all patients in terms of thickness, texture and color and flaps provided stable coverage throughout the follow-up period. 


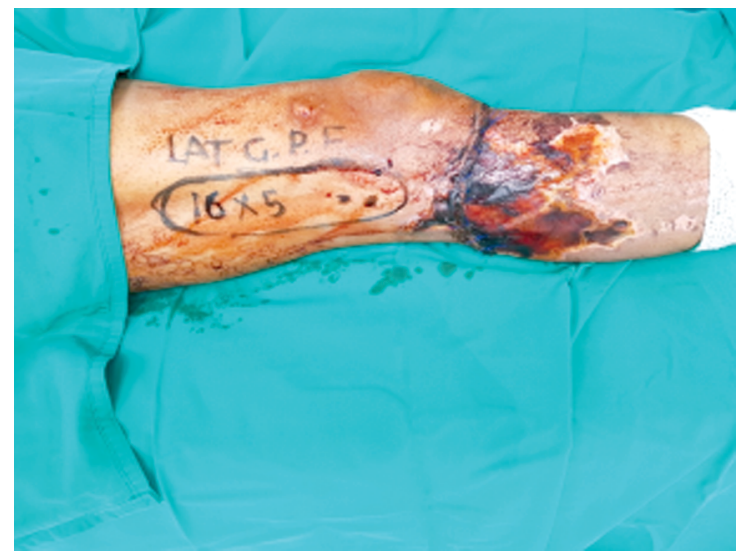

Fig. (2-A): Preoperative photo. Male patient 33 years with post RTA skin necrosis of the upper lateral Rt leg. An SLGA perforator flap, $16 \times 5 \mathrm{~cm}$ was marked. Doppler-detected 2 perforators marked by 2 dots at flap pivot point.

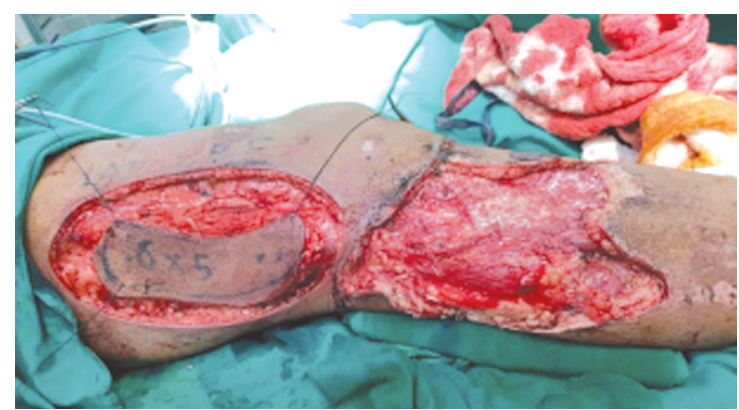

Fig. (2-C): The SLGAp flap was totally islanded, hinged only by the main perforator, in preparation for propeller rotation.

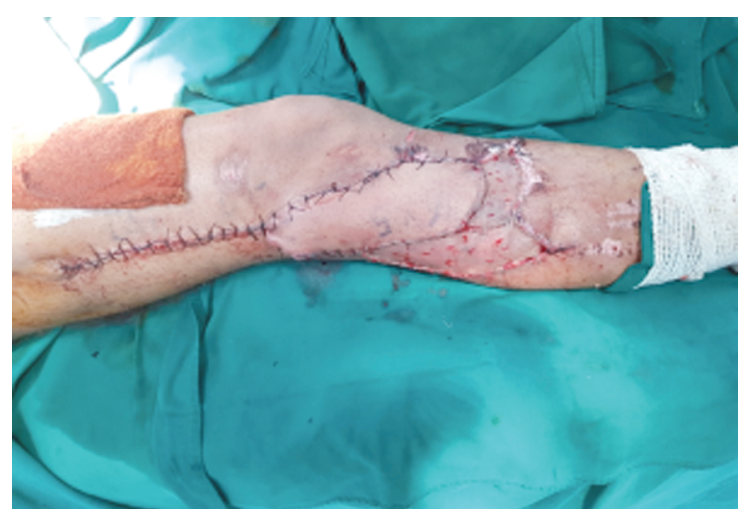

Fig. (2-E): Final appearance at end of procedure.

Fig. (2-G): Final appearance after secondary skin grafting with accepted functional outcome.

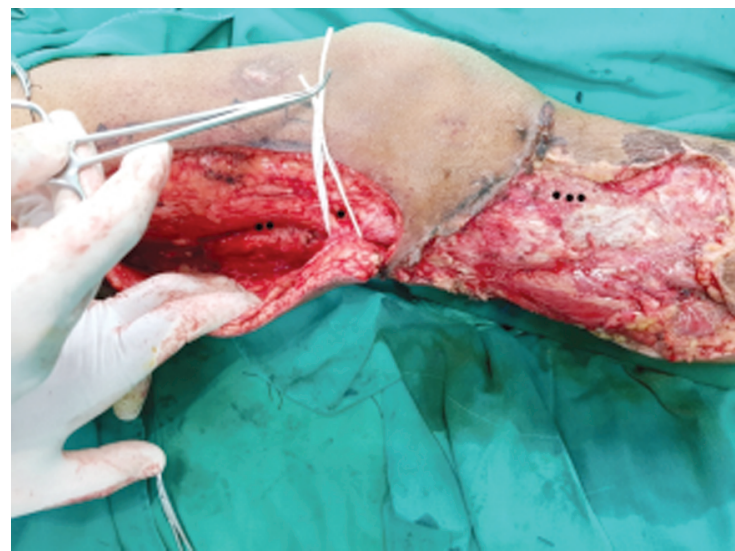

Fig. (2-B): Debridement of necrotic skin resulted in exposure of tibial tuberosity marked by 3 black dots. The SLGAp flap was dissected subfascially and the main perforator, marked by black dot, was isolated. The intermuscular septum marked by 2 black dots.

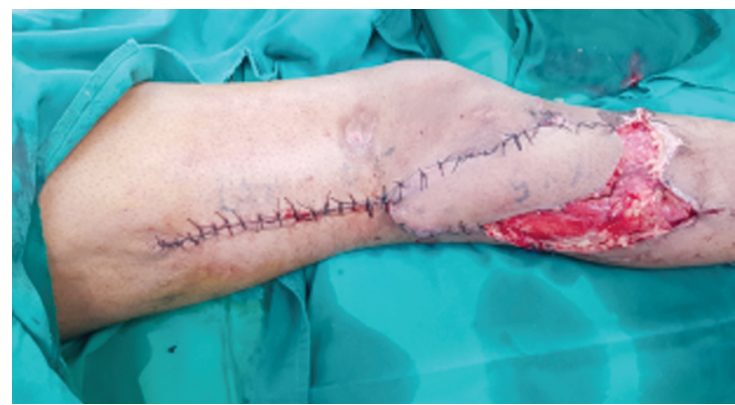

Fig. (2-D): SLGAp flap was rotated in a propeller fashion to cover tibial tuberosity and donor site closed primarily and remaining raw area was skin-grafted.

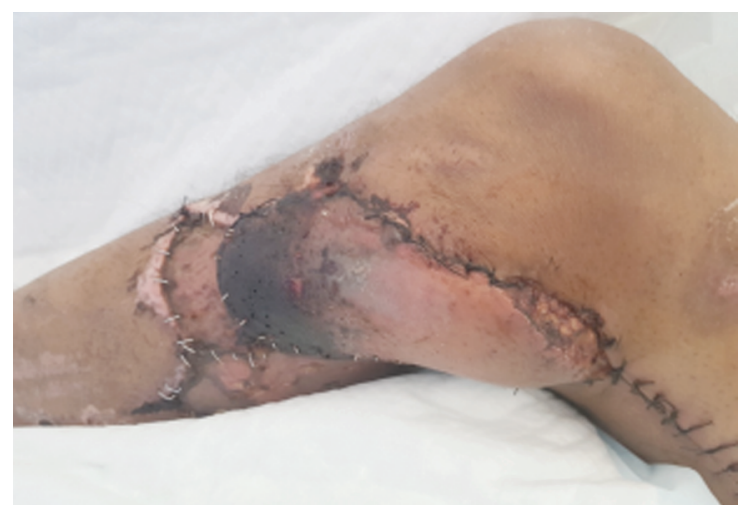

Fig. (2-F): Full thickness necrosis of distal third of the flap due to venous congestion. Debridement was done followed by skin grafting.

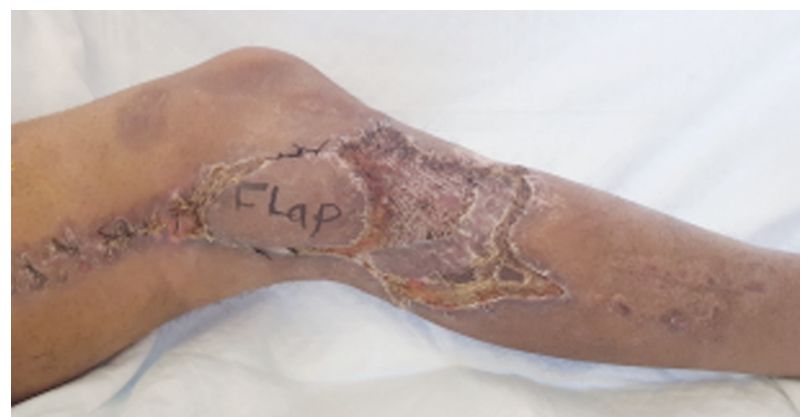




\section{DISCUSSION}

Soft tissue defects around the knee represent a reconstructive challenge. These defects may occur due to different etiologies, as traffic accidents, shotgun, contracture release...etc. There are many reconstructive options for covering these soft tissue defects, including several muscle flaps, fasciocutaneous flaps, and free flaps. Each option has its advantages and disadvantages [1-10].

Hayashi A. and Maruyama Y. had done 3 cases of SLGA perforator flap with direct closure of the donor site. All the three flaps survived, except superficial tip necrosis $3 \mathrm{~cm}$ in width in 1 flap and healed well by conservative therapy [12].

Taniguchi Y. and his colleagues had done a one case of SLGA perforator flap for treatment of skin necrosis after total knee arthroplasty. The flap survived completely and good clinical outcome was obtained [15].

Saint-Cyr M., et al., had done 2 cases of SLGA perforator flaps. The 2 flaps survived well, except for minor delayed tip healing and widening of the donor site scar [16].

Wiedner M. and her colleagues had done 6 SLGA perforator flaps in their study. There was no flap loss in their patients. Three patients had partial flap loss at distal tip of the flap. Two of these patients were treated with skin graft and one patient healed conservatively. Primary donor site closure was possible in all their patients, with no late complications either in the flap area or in the donor site region [17].

Zhihua Li BS, and his colleagues had done 5 SLGAp flaps in their study. All the flaps survived well without adverse events. Early, the flaps were locally bloated, but later on, the appearance of the flap was good and close to normal. All their patients were satisfied with flap appearance and functional recovery [18].

The results of our study were more or less comparable to these studies. We had found that the superior lateral genicular artery perforator flap is a versatile and valuable option for coverage of defects of knee and popliteal fossa. Donor site morbidity is minimal with no functional deficit. The anatomy of the SLGA perforators is consistent and reliable. The SLGA perforator flap is a good option for coverage of defects around the knee due to the above mentioned attributes.

Limitations of the present study include the limited number of cases, the natural limitations of a retrospective review, and subjective evaluation of the functional and aesthetic outcomes by measuring patient satisfaction only. Further studies with large number of patients are required to objectively evaluate aesthetic outcome by numerical grading scale and evaluate functional outcome by measuring degree of flexion/extension deficit of the knee joint pre and postoperatively, with statistical analysis of the results. Despite these limitations, we feel that this flap has the potential to augment our armamentarium of tools available for surgical coverage of around knee soft tissue defects, especially if the gastrocnemius (workhorse) flap is not available for use.

\section{Conclusion:}

The superior lateral genicular artery perforator flap is a valuable option for reconstruction of soft tissue defects around the knee or popliteal fossa, especially when the gastrocnemius muscle is not available for use. The anatomy of the SLGA perforators is consistent and reliable. The flap is thin, pliable, and provides stable coverage, with adequate match of characters to surroundings. Donor site morbidity is minimal with no functional deficit.

\section{REFERENCES}

1- Elsahy N.I.: Cover of the exposed knee joint by the lateral head of the gastrocnemius. Br. J. Plast. Surg., 31: 136-7, 1978.

2- Feldman J.J., Cohen B.E., May Jr. J.W.: The medial gastrocnemius myocutaneous flap. Plast. Reconstr. Surg., 61: 531-9, 1978.

3- Swartz W.M., Ramasastry S.S., Mc Gill J.R., et al.: Distally based vastus lateralis muscle flap for coverage of wounds about the knee. Plast. Reconstr. Surg., 80: 255-56, 1987.

4- Petty C.T. and Hogue Jr. R.J.: Closure of an exposed knee joint by use of a Sartorius muscle flap: Case report. Plast. Reconstr. Surg., 62: 458-61, 1978.

5- Ponten B.: The fasciocutaneous flap: Its use in soft tissue defects of the lower leg. Br. J. Plast. Surg., 34: 215-20, 1981.

6- Lin S.D., Lai C.S., Chiu Y.T., et al.: Adipofascial flap of the lower leg based on the saphenous artery. Br. J. Plast. Surg., 49: 390-5, 1996.

7- Kasabian A.K., Colen S.R., Shaw W.W., et al.: The role of the microvascular free flaps in salvaging below-knee amputation stumps: A review of 22 cases. J. Trauma, 31: 495-500, 1991.

8- Mc Craw J.B., Fishman J.H. and Sharzer L.A.: The versatile gastrocnemius myocutaneous flap. Plast. Reconstr. Surg., 62: 15-23, 1978.

9- Nahabedian M.Y., Morat M.A., Orlando J.C., et al.: Operative management and outcome of complex wounds following total knee arthroplasty. Plast. Reconstr. Surg., 104: 688-97, 1999. 
10- Zhang G.: Reversed anterolateral thigh island flap and myocutaneous flap transplantation. Chinese Journal of Stomatology, 70: 676-8, 1990.

11- Laitung J.K.: The lower postrolateral thigh flap. Br. J. Plast. Surg., 42: 133-9, 1989.

12- Hayashi A. and Maruyama Y.: The lateral genicular artery flap. Ann. Plast. Surg., 24: 310-17, 1990.

13- Spokevicius S. and Jankauskas A.: Anatomy and clinical applications of composite cutaneo-subcutaneous flap based on the lateral superior genicular vessels. J. Reconstr. Microsurg, 11: 15-20, 1995.

14- Li X., Huang S., Suj, et al.: Investigation of a retrograded supragenicular flap for treatment of scarring contraction of popliteal fossa. Chinese Journal of Plastic Surgery and Burns, 18: 343-5, 2002.
15- Taniguchi Y., Kitano T., Shimoe T., et al.: Superior lateral genicular artery flap for coverage of a soft tissue defect after total knee arthroplasty. J. Reconstr. Microsurg, 25: 479-82, 2009.

16- Saint-Cyr M., Wong C., Nguyen A.T., et al.: Lateral supragenicular pedicle perforator flap: Clinical results and vascular anatomy. J. Plast. Reconstr. Aesth. Surg., 64: 381-85, 2011.

17- Wiedner M., Koch H. and Scharnagl E.: The superior lateral genicular artery flap for soft-tissue reconstruction around the knee: Clinical experience and review of the literature. Ann. Plast. Surg., 66 (4): 388-92, 2011.

18- Zhihua Li B.S., Pandeng Li B.S. and Qian Tan: Reconstruction of soft tissue defects around the knee with pedicled perforator flaps. Ann. Plast. Surg., 81 (4): 46267, 2018. 
